\title{
Enhancing the in vitro Antioxidant Capacities via the interaction of amino acids
}

\author{
Jingbo Liu, Deju Zhang, Yinshi Zhu, Yizhen Wang, Shucheng He, Ting Zhang* \\ Jilin Key Laboratory of Nutrition and Functional Food, Jilin University, Changchun 130062, P.R. China.
}

\section{A B S T R A C T}

\begin{abstract}
In this article, antioxidant capacities of 20 amino acids were quantitatively determined via methodology of mixingone amino acid with the others and using four antioxidant assays to understand the synergistic effects of the individual amino acid contributing to the total antioxidant capacity. Some of the individual amino acids and their mixtures showed their characteristics of antioxidant capacities, and antioxidant peptides showed different results in different experiments. From the ABTS assay study, it was found that nine of 20 amino acids had antioxidant capacities, and most of their mixtures had synergetic effects, Moreover, an oxygen radical absorbance capacity assay study was conducted, and the results aligned well with the ABTS study. However, the DPPH and FRAP assay studies, indicated that cysteine was the only amino acid having antioxidant effect in 20 amino acids. The result showed that the combinations of high antioxidant amino acids and weak antioxidant amino acids or non-antioxidant amino acids led to an obvious increase in antioxidant capacity compared with the theoretical antioxidant capacity.
\end{abstract}

Keywords: ABTS, amino acid, antioxidant capacity, DPPH, FRAP, ORAC

\section{INTRODUCTION}

It was reported that several serious diseases, such as cancers and diabetes, were all related to the excessive oxidation in human body induced by free radicals(Saoudi et al., 2016). Free radicals like reactive oxygen species (ROS), reactive nitrogen species (RNS) and reactive sulphur species (RSS) (Carocho and Ferreira 2013) are often associated with diseases (Robbins et al., 2016). For reducing the toxicity of free radicals and boost the body's natural defences, antioxidants are used widely nowadays (Al-Gubory et al., 2016).Chemical synthetic antioxidants may have damage to tissues and organs(Carocho et al., 2015), which drives the development of natural antioxidants to prevent many chronic diseases. Recently, new natural antioxidants, the biological activities of the amino acids have attracted people'sattention, as they have proven effectiveness in the prevention of biochemical and physiological lesions(Cai et al., 2016).A great number of antioxidant peptides have been identified,andthe antioxidant properties of some amino acids were firstly reported by Marcuse(Marcuse 1960). Antioxidant actions of peptides are associated with their amino acid constituents (Ren et al., 2014).
On the other hand, scientists found that several amino acids can be antioxidant and prooxidant activity compounds (Vavrusova et al., 2015).Nevertheless, a single antioxidantamino acid cannot replace the combination of natural phytochemicals in fruit to achieve health benefits(Liu 2004).It is proved that the benefit is attributed to the complex mixture of phytochemicalspresented in whole foods(Liu 2003).

A synergistic effect is the action of chemicals is individually or in combination (Yang and Liu, 2009). Antioxidant synergism has been strengthened with different compounds such as folic acid and vitamin B-12 (Bhattacharjee et al., 2016), vitamin $\mathrm{E}$ and $\beta$-carotene (Palozza and Krinsky 1992), catechin and malvidin 3-glucoside(Rossetto et al., 2002) and tea polyphenols and vitamin E(Nagaoka et al., 2016). Therefore antioxidant synergistic effects of different amino acids might have a greater effect than that ofthe single antioxidant amino acid and there were only limited reports available on this topic.

The present research aims to investigate the antioxidant capacities and synergistic effects of the 20 amino acids via different antioxidant assays.

\footnotetext{
${ }^{*}$ Corresponding author:

Dr. Ting Zhang, ; Tel.: +86-431-8783-6531; Fax: +86-431-8783-6391. E-mail address: tingzhang@jlu.edu.cn
}

Received: 1 September 2017; $\quad$ Accepted: 17 February 2018 


\section{MATERIALS AND METHODS}

\section{ABTS Radical scavenging activity assay}

The ABTS radical-scavenging capacity was determined according to the method described by Re et al. (Re et al., 1999) and Balcerczyk et al. (Balcerczyk et al., 2007) with the following modifications. The ABTS radical solution was prepared by the following procedure:

1) Mix $10 \mathrm{ml}$ of $7 \mathrm{mM}$ ABTS radical solution with $10 \mathrm{ml}$ of $2.45 \mathrm{mM}$ potassium persulfate solution to obtain mixture A.

2) Leave the mixture $A$ in the darkness for $12-16$ h to allow the development of radicals.

3) Dilute the mixture $A$ with $0.1 \mathrm{mM}$ Phosphate buffer ( $\mathrm{pH} 7.4$ ) to reach the UV-Vis absorbance of $0.7( \pm 0.02)$ at $734 \mathrm{~nm}$ wavelength before the usage. An aliquot of the sample solution $(20 \mu \mathrm{L})$ at different concentrations was added to the diluted ABTS.+ solution $(180 \mu \mathrm{L}$, followed by 7 -min incubation at room temperature. After then the absorbance of the mixture at $734 \mathrm{~nm}$ was measured. The ABTS scavenging activity was calculated as following:

ABTS radical

scavenging $=\left(\mathrm{A}_{\text {control }}-\mathrm{A}_{\text {sample }}\right) / \mathrm{A}_{\text {control }} \times 100$

activity $(\%)$

\section{Ferric reducing antioxidant power (FRAP) assay}

The FRAP assay was introduced by Benzie (Benzie and Strain 1996), and values were expressed as mmol Fe ${ }^{2+} / \mathrm{mg}$ of sample. All the treatment groups were measured in triplicate.

\section{Oxygen radical absorbance capacity (ORAC) assay}

The oxygen radical absorbance capacity (ORAC) assay using fluorescein as probe was determined according to the method of Ou et al. (Ou et al., 2003) and was slightly modified by Davalos et al. (Davalos et al., 2004).

\section{DPPH radical-scavenging assay}

The DPPH radical-scavenging capacity was determined according to the method described by Hatano et al. (Hatano et al., 2006).

\section{Experimental design and synergistic antioxidant capacity evaluation}

We prepared 20 kinds of amino acids and measured the antioxidant capacites of them in a series of concentrations (2.0 $\mu \mathrm{M}-10.0 \mathrm{mM}$ final concentration), then we sift through the antioxidant amino acids and combine high antioxidant amino acids with high antioxidant amino acids, weak antioxidant amino acids or non-antioxidant amino acids, their synergistic antioxidant activity was measured by DPPH, ABTS, ORAC, FRAP. In DPPH assay, the concentration of non-antioxidant amino acids and Cys are $100 \mu \mathrm{M}$ and $300 \mu \mathrm{M}$, respectively. In ABTS assay, the concentration of high antioxidant amino acids, weak antioxidant amino acids and non-antioxidant amino acids are $2 \mu \mathrm{M}, 2 \mathrm{mM}$ and $100 \mu \mathrm{M}$, respectively. In ORAC assay, the concentration of high antioxidant amino acids, weak antioxidant amino acids and non-antioxidant amino acids are $10 \mu \mathrm{M}, 2 \mathrm{mM}$ and $100 \mu \mathrm{M}$, respectively. In FRAP assay, the concentration of non-antioxidant amino acids and Cys are $100 \mu \mathrm{M}$ and $300 \mu \mathrm{M}$, respectively. All assays were carried out in triplicate.

In order to summarize the interaction between two antioxidant samples, we define antagonism as negative synergistic effect (summated $>$ measured) and the synergy as positive synergistic effect between the two substances (summated $<$ measured)

measured $=\frac{M_{1}+M_{2}}{2}$

$M_{1}$ means the antioxidant capacity of peptide 1 alone,

$\mathrm{M}_{2}$ means the antioxidant capacity of peptide 2 alone,

Summated means the antioxidant capacity of combined peptide 1 and peptide 2 .

In order to summarize the interaction between antioxidant samples and non-antioxidant samples, we measured the antioxidant capacity of the sample at half concentration, $A_{1}$, and compared it with the antioxidant capacity of the combined antioxidant samples and non-antioxidant samples, $A_{2}$, if $A_{1}>A_{2}$, there was antagonism effect between the two samples. However if $A_{1}<A_{2}$, there was synergistic effect between the two samples.

\section{Data analysis}

Analysis of variance (ANOVA) was performed by using the SPSS 19.0 software at a significant level of $\mathrm{P}<0.05$. All data were expressed as the mean \pm SD $(n=3)$.

\section{RESULTS}

\section{$A B T S$ radical scavenging activity results}

ABTS, which has been widely used in the measurement of total antioxidant activity, is a kind of stable free radical cation that is extensively applicable to monitor the antioxidant capacity of hydrophilic antioxidant compounds.

The result of ABTS assay which displayed nine of 20 amino acids examined had ABTS $^{+}$radicals abilities of varying degree. Results obtained from the amino acids under similar conditions were shown in Fig. 1. According 
to the rate of antioxidant capacity, we divided all of them into three groups. From the reliability of the data given in Fig. 1, we classified cysteine, tyrosine and tryptophan as Group 1 due to its high antioxidant capacity. Specifically, the reactivity of these three amino acids, under the same condition, decreased in the following order:

Tyr $>$ Trp $>$ Cys

On the other hand, there were also some amino acids with much lower antioxidant capacities but cannot be ignored, so it was very easy to classify them together as Group 2. Fig. 1 showed that the rank order of other amino acids according to their antioxidant effects was:

Lys $>$ His $>$ Arg $>>$ Val $>$ Phe $>$ Met

And all other amino acids did not show any activities.

What is more, the simple combination of two amino acids was used to evaluate the effect of amino acid mixtures on total antioxidant capacity. And the synergistic effects were calculated by the direct comparison methodology which

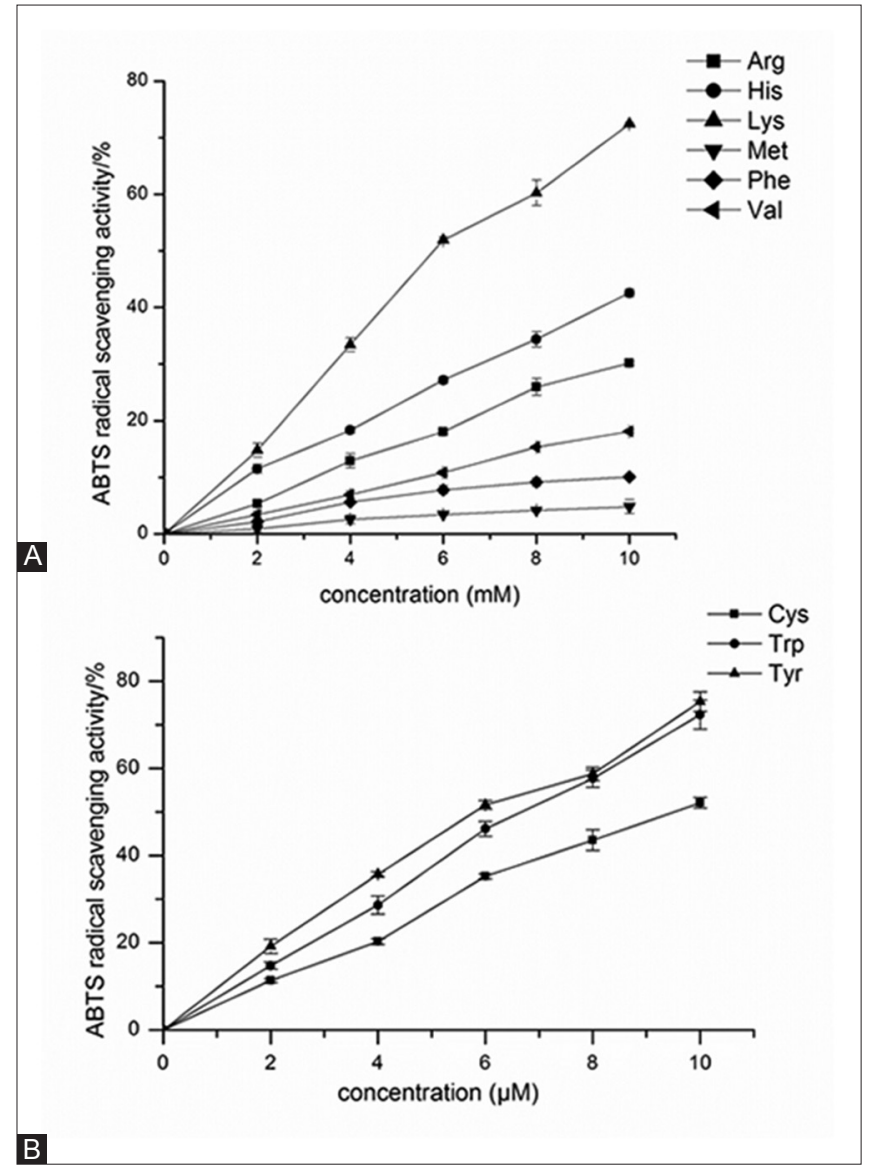

Fig 1. (A) ABTS radical scavenging activity of amino acids (Arg, His, Lys, Met, Phe, Val) incubated in solutions (PBS) at various concentrations for $2 \mathrm{mM}, 4 \mathrm{mM}, 6 \mathrm{mM}, 10 \mathrm{mM}$. (B) ABTS radical scavenging activity of amino acids (Cys, Trp, Tyr) incubated in solutions (PBS) at various concentrations for $2 \mu \mathrm{M}, 4 \mu \mathrm{M}, 6 \mu \mathrm{M}, 8 \mu \mathrm{M}$. Data are means \pm standard deviation, ${ }^{*} p$ indicate the statistically significant differences $(p<0.05)$ between different treatments, ${ }^{* *} p$ indicate the statistically significant differences $(p<0.01)$ between different treatments. was reported by Liu (Di Liu 2016). High antioxidantive amino acids were combined with weak antioxidantive amino acids or non-antioxidantive amino acids, and these combined amino acids showed different antioxidant activity compared with individual amino acids. High antioxidant amino acids had similar antioxidant capacity with weak antioxidant amino acids when its concentration was $2 \mu \mathrm{M}$ and $2 \mathrm{mM}$, respectively, so we combined them at different concentration levels to make it easier to be compared. Each amino acids of Group 1 was combined with the other eleven amino acids without antioxidant capacities. To assess how amino acid interactions contribute to the total antioxidant capacity in the model mixture of two amino acids, each antioxidantive amino acid was added to the other amino acid and then antioxidant capacity was analysed by the ABTS assay.

Fig. 2 showed the effect of the addition of antioxidantive amino acids to the other antioxidantive amino acids and non-antioxidantive amino acids on total antioxidant capacity. Fig. 2A, Fig. 2B, Fig. 2C showed comparison between antioxidantive amino acids and the combination of antioxidantive amino acids and non-antioxidantive amino acids, and Fig. 2D Fig. 2E, Fig. 2F showed the antioxidant activity of combined amino acid in the theoretical value and experimental value. The addition of Tyr to Asp, Gln, Glu, Ile, Gly, Asn, Leu, Pro, Ser, Thr, His, Lys, Arg, Cys to Ala, Asp, Gln, Ile, Gly, Asn, Ser, Arg, Lys, and Tyr to Ala, Gln, Glu, Gly, Asn, Leu, Pro, Ser, Thr, Lys, Arg led to an increase of antioxidant capacity compared with the theoretical antioxidant capacity, while the addition of Cys

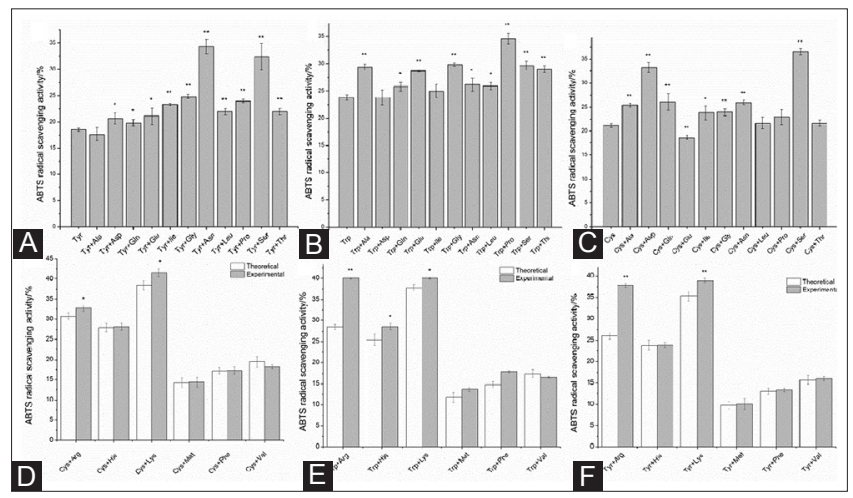

Fig 2. Antioxidant capacity was estimated by ABTS assay, the results are expressed as the mean \pm SEM for each group $(n=6)$ -high antioxidant capacity amino acids, non-antioxidant amino acids combined with high antioxidant capacity amino acids, weak antioxidant capacity amino acids and high antioxidant capacity amino acids, (A) Tyr and Tyr combined with non-antioxidant amino acids. (B) Trp and Trp combined with non-antioxidant amino acids. (C) Cys and Cys combined with non-antioxidant amino acids. (D) high antioxidant capacity amino acids combined with weak antioxidant capacity amino acids. *p indicate the statistically significant differences $(p<0.05)$ between different treatments, ${ }^{* *} p$ indicate the statistically significant differences $(p<0.01)$ between different treatments. 
to Glu led to a decrease of the measured total antioxidant capacity.

The results also suggested that there was no synergistic effect between each pair of amino acids in Group 1 and Group 2, between Cys and Met, Phe, His, Val, Trp to Met, Phe, Val, and between Tyr to His, Phe, Met, Val on total antioxidant capacity of the mixture of two amino acids (data not shown).

\section{Ferric reducing antioxidant power}

The FRAP assay, which is a method of assessing "antioxidant power", is an automated test about measuring the decreasing ferric ability of plasma. The FRAP assay offers a rapid technique for screening radical scavenging activity of specific compounds (Benzie and Strain 1996). Cys was the amino acid which shows the highest FRAP value, with $36.12 \mu \mathrm{M} \mathrm{TE} / \mu \mathrm{M}$ at the concentration of $100 \mu \mathrm{M}$. And the FRAP ability showed the upward trend when the concentration of amino acid increased.

There was also a synergistic effect between Cys and any other amino acids. Fig. 3A shows that the addition of Cys to Leu, Met, Thr, Tyr and Val caused an obvious increase of antioxidant capacity compared with the theoretical antioxidant capacity.

\section{DPPH radical-scavenging activity}

DPPH radical scavenging activity estimation is extensively applicable to monitor the measurement of antioxidant capacity and offers a putative index of antioxidant. Unlike the result of the ABTS radical scavenging activity, the result appears that only examined Cys had antioxidant capacity. The results of DPPH and FRAP assays showed no differences in determinations.

Then, we detected the total antioxidant capacities between two kinds of amino acids with simple combination of Cys and every non-antioxidant amino acid. It was apparent that the addition of Cys to Ala, Asp, Glu, His and Asn caused an obvious increase in antioxidant capacity compared with the theoretical antioxidant capacity. While the addition of Cys to Ser and Thr caused a decrease in antioxidant capacity during the process of DPPH scavenging activity. It might be the -OH in the side chain groups of Ser and Thr residues that play a role in the synergistic or antagonistic effect of combined amino acids, thereby reducing the hydrogen supply capacity of the amino acid. However, antioxidant reaction sometimes might be very complicated, it is difficult to explain different phenomenon from the physical and chemical properties of amino acids.

We can conclude from the two figures that there were significant similarities between the results of FRAP assay and DPPH assay. Cys was the amino acid which owned

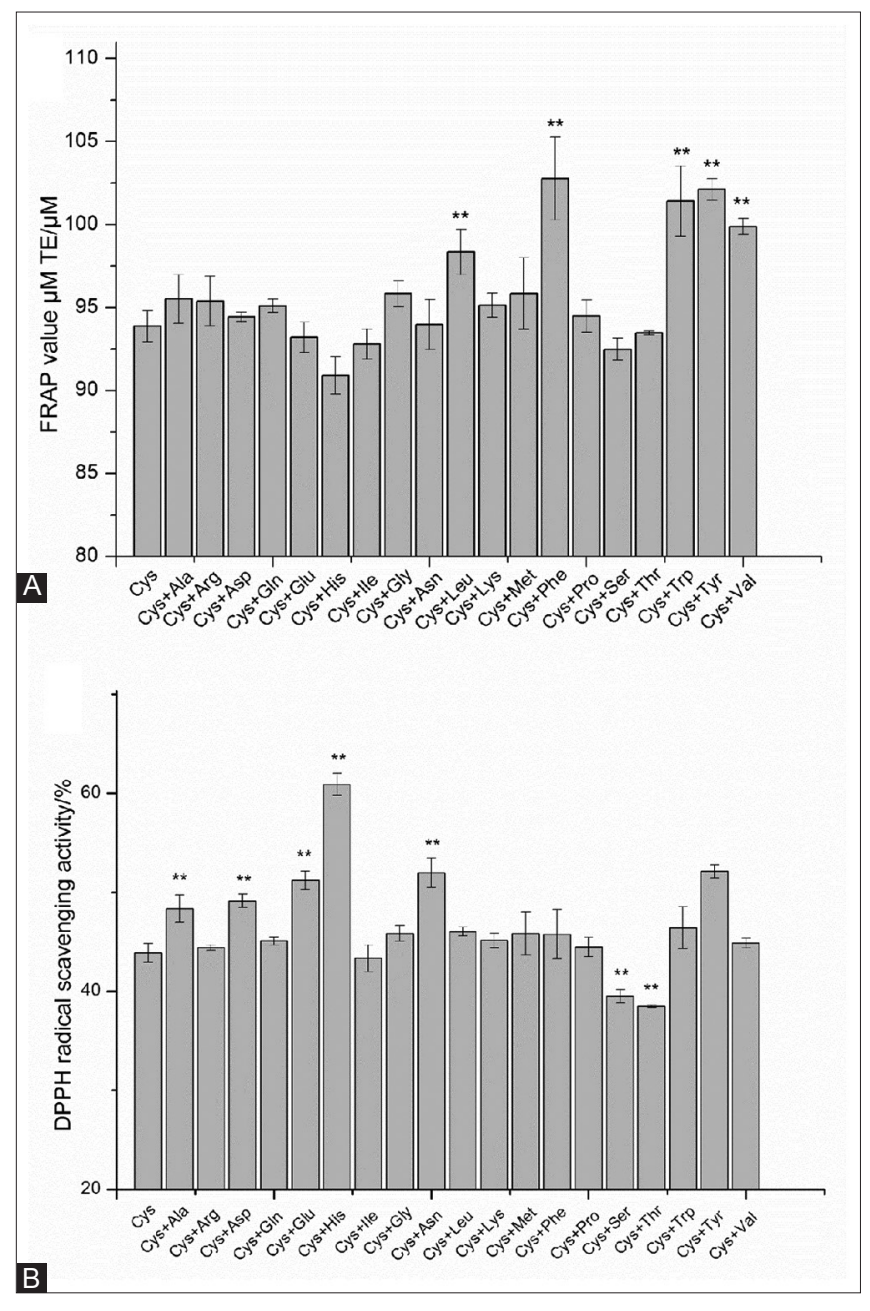

Fig 3.(A) Antioxidant activities and synergistic effect of all amino acids determined by FRAP assay, the results are expressed as the mean \pm SEM for each group $(n=6)$-high antioxidant capacity amino acids (Cys), non-antioxidant amino acids combined with high antioxidant capacity amino acids (Cys). (B) Antioxidant activities and synergistic effect of all amino acids determined by DPPH assay, the results are expressed as the mean \pm SEM for each group $(n=6)$-high antioxidant capacity amino acids (Cys), non-antioxidant amino acids combined with high antioxidant capacity amino acids (Cys). ${ }^{*}$ p indicate the statistically significant differences $(p<0.05)$ between different treatments, ${ }^{* *} p$ indicate the statistically significant differences $(p<0.01)$ between different treatments.

antioxidant capacity and had synergistic effect with other non-antioxidantive amino acid.

\section{Oxygen radical absorbance capacity assay}

The result of ORAC assay which showed the nine of 20 amino acids examined had Oxygen radical absorbance capacity. ORAC values from each of them were shown in Fig.4, which also showed the rank order of nine amino acids according to their antioxidant effects:

Tyr $>$ Met $>$ Trp $>$ Cys $>$ His $>>$ Lys $>$ Leu $>$ Phe $>$ Arg. And the other amino acids did not show any activities.

What is more, Fig.4 also presented that the antioxidant capacity was up with linear when the concentration of the 
amino acid increased, and there were significant similarities in the results of ABTS assay and ORAC assay.

Various concentrations of amino acids owned moderate oxygen radical absorbance capacities. To assess how amino acid interactions contribute to total antioxidant capacity of the model mixture of two amino acids, the ORAC assay was applied.

Fig. 5 showed the effect of the addition of antioxidant amino acid to the other antioxidant amino acids on total antioxidant capacity. The addition of Cys to His, Met, Trp, His to Met, Trp, Arg to Cys, His, Met, Trp, Tyr, Leu to Cys, His, Met, Trp, Tyr, Lys to His, Met, Trp, Tyr, Phe to Cys, Met, Trp caused an increase of antioxidant capacity compared with the theoretical antioxidant capacity.

A
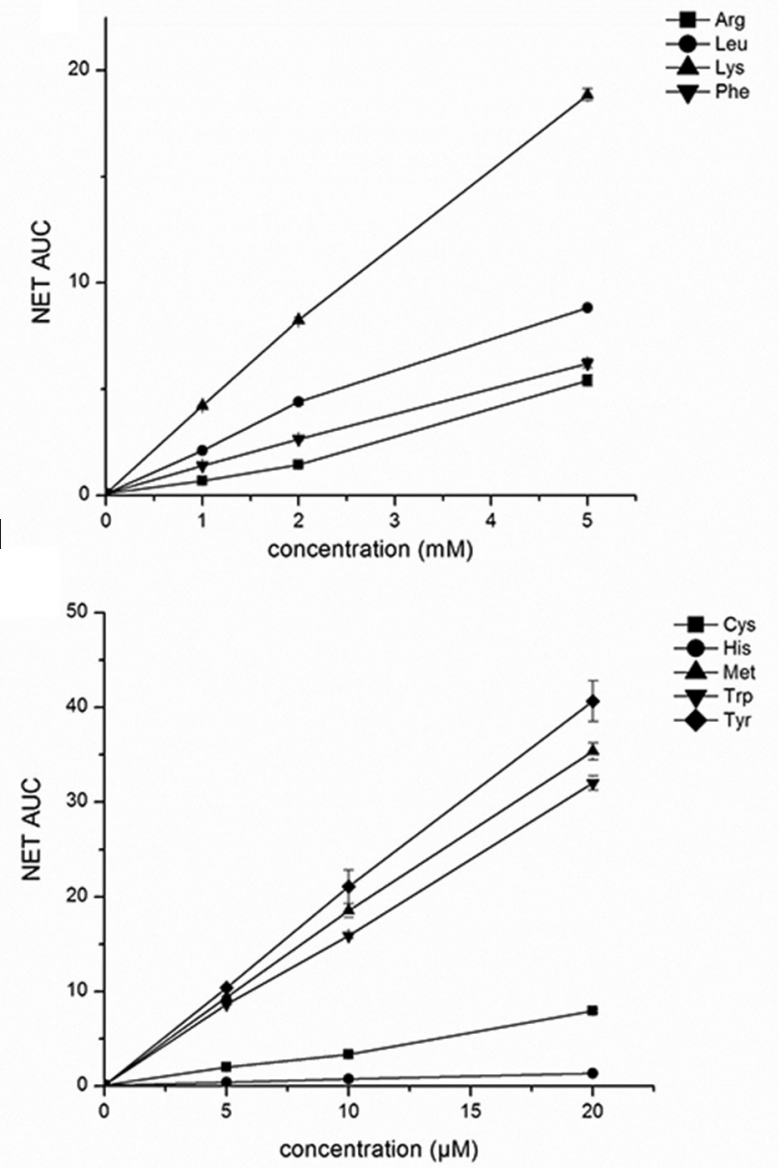

B

Fig 4. (A) Oxygen radical absorbance capacity of amino acids (Arg, Leu, Lys, Phe) incubated in solutions (PBS) at various concentrations for $1 \mathrm{mM}, 2 \mathrm{mM}, 5 \mathrm{mM}$. (B) Oxygen radical absorbance capacity of amino acids (Cys, His, Met, Trp, Tyr) incubated in solutions (PBS) at various concentrations for $5 \mu \mathrm{M}, 10 \mu \mathrm{M}, 20 \mu \mathrm{M}$. Data are means \pm standard deviation, ${ }^{*} p$ indicate the statistically significant differences $(p<0.05)$ between different treatments, ${ }^{* *} p$ indicate the statistically significant differences $(p<0.01)$ between different treatments.
Fig.6 showed the effect of the addition of one antioxidant amino acid to the other non-antioxidant amino acids on the total antioxidant capacity. The addition of Cys to Ala, Gly, Phe, Ser and Thr, His to Ala, Asp, Gln, Glu, Ile, Gly, Asn, Phe, Ser, Thr and Val, Met to Ala, Asp, Gln, Glu, Ile andVal, Trp to Ala, Gln, Glu, Asn, Phe and Thr, and Tyr to Asp, Gln, Glu, Ile, Asn, Ser and Thr caused an increase in antioxidant capacity compared with the theoretical antioxidant capacity.

\section{DISCUSSION}

Damgaard et al. (Damgaard et al., 2015)found that the ABTS radical scavenging capacity was correlated with the

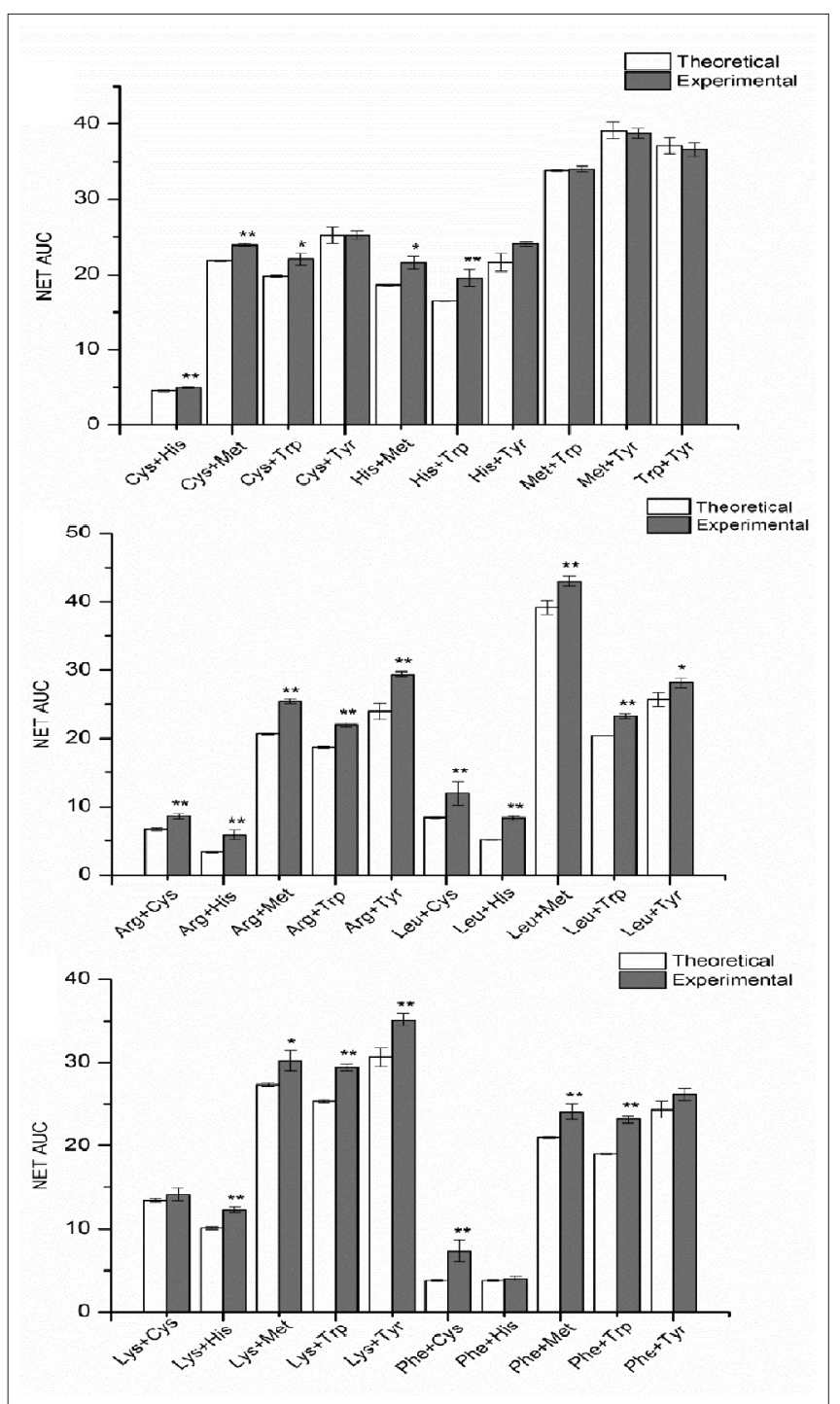

Fig 5. Synergistic effect of antioxidant amino acid determined by ORAC assay, the results are expressed as the mean \pm SEM for each group $(n=6)$-weak antioxidant amino acids combined with high antioxidant capacity amino acids, high antioxidant capacity amino acids combined with high antioxidant capacity amino acids, *p indicate the statistically significant differences $(p<0.05)$ between different treatments, ${ }^{* *} p$ indicate the statistically significant differences $(p<0.01)$ between different treatments. 


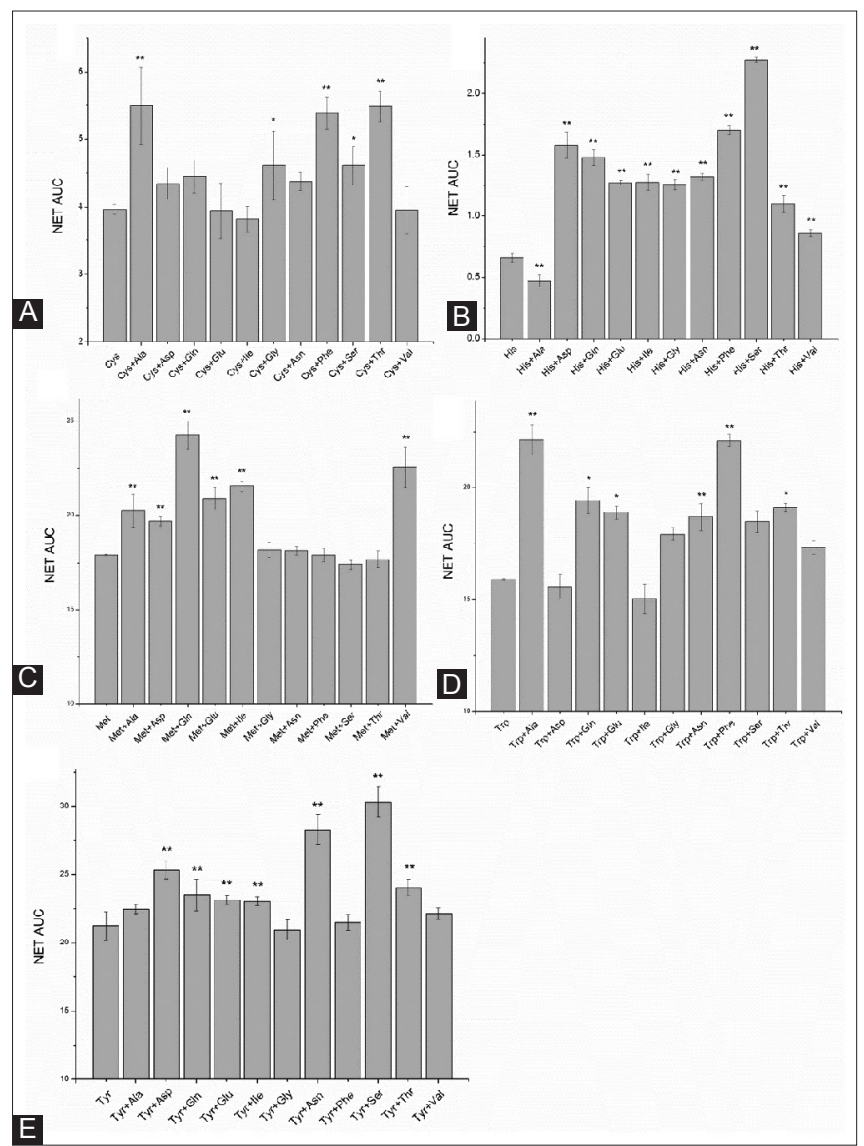

Fig 6. Synergistic effect of antioxidant amino acid and non-antioxidant amino acid determined by ORAC assay. the results are expressed as the mean \pm SEM for each group $(n=6)$-non-antioxidant amino acids combined with high antioxidant capacity amino acids, (A) Cys and Cys combined with non-antioxidant amino acids. (B) His and His combined with non-antioxidant amino acids. (C) Met and Met combined with nonantioxidant amino acids. (D) Trp and Trp combined with non-antioxidant amino acids. (E) Tyr and Tyr combined with non-antioxidant amino acids. ${ }^{*} p$ indicate the statistically significant differences $(p<0.05)$ between different treatments, ${ }^{* *} p$ indicate the statistically significant differences $(p<0.01)$ between different treatments.

content of Trp, Tyr, Met and Arg. Besides Wiriyaphan et al. (Wiriyaphan et al., 2013) also found that peptides which contained Tyr, Trp, Cys and His residues presented high ABTS radical scavenging activity. From this study, we found that onlynine amino acids (Tyr, Trp, Cys, Lys, His, Arg, Val, Phe and Met) showed ABTS radical scavenging capacity, and there were synergistic antioxidant effectson amino acids detected by the direct comparison methodology which was reported by Liao (Kuei-long Liao 2000). Wiriyaphan et al. (Wiriyaphan et al., 2013)determined the antioxidant activity of peptides derived from the product of threadfin bream surimi, and the results demonstrated that the decreasing ferric antioxidant power of peptide was closely related to the amounts of Trp, Met and Cys. Zheng et al. (Zheng et al., 2016)discovered that the antioxidant behaviours of peptides strongly depended on the compositions and reactionsof the amino acid residues, and the Cys could effectively scavenge DPPH radicals, which was consistent with our result, while Tyr, Trp and Gly showed a little or even no DPPH radical scavenging activities. Triantis et al. (Triantis et al., 2007)found that Cys contained one free-SH group, this free -SH group had strong electron-donating ability and hydrogen-donating ability, this may be the reason why cysteine has high antioxidant capacity. Met is prone to oxidation to Met sulfoxide and Cys donates the sulfur hydrogen, and thus Met also have a certain degree of free radical scavenging ability (Hernandez-ledesma et al., 2005). Phe and Lys also have weak free radical scavenging ability in ORAC and ABTS, which may be related to the supply capacity of benzene ring and $-\mathrm{NH}_{2}$ on its side chain groups. Garrett et al. (Garrett et al., 2014)examined 20 amino acids by using the ORAC assay, the results indicated that Try, Tyr and Met had the highest ORAC values, Asp, and Cys, His, Lys, Leu, Phe and Arg got weak ORAC values, while Thr, Glu, Asp, Iso, Leu, Glu, Ser, Lys, Val, Ala and Gly had non-ORAC values. Wang et al. (Wang et al., 2016a) found that the samples which contained rich acidic and hydrophobic amino acids revealed high antioxidant capacities in TEAC and ORAC assay, and these amino acids had strong antioxidant capacities in our research as well. Hye Jung et al. (Ka et al., 2016) tested eight amino acids and discovered that Cys showed the highest antioxidant capacity during the different methods of in vitro antioxidant determination. However, it did not show high antioxidant capacity in $\mathrm{O} / \mathrm{W}$ emulsions. In the present study, there was an obvious difference between the results of ABTS/ORAC and FRAP/DPPH. And the results of FARP were similar to those of DPPH. Cys was the only amino acid to show the antioxidant capacity, while the others had a little or even no antioxidant capacities. The results of ORAC were also similar to those of ABTS but with slight difference. Tyr, Trp and Cys showed high antioxidant capacities in ORAC and ABTS assay respectively, while Met and His showed high antioxidant capacities in ORAC assay but weak ones in ABTS assay. Arg, Lys and Phe showed weak antioxidant capacities in ORAC and ABTS assay. Moreover Val had weak antioxidant capacities in ABTS assay, but it didn't show any antioxidant capacities in ORAC assay. By contrast, Leu had weak antioxidant capacities in ORAC assay, but it didn't exhibit any antioxidant capacities in ABTS assay.

Many antioxidants are able to react rapidly with the ROO• in ORAC, but react very slowly or even non-react with $\mathrm{DPPH} \cdot \mathrm{DPPH} \bullet$ is a kind of stable free radical and has weak reactivity, and therefore it can only react with Cys or Cysteine residues, which containing stronger reducing power, the rest of the amino acids are not equal to restore it to DPPHH. While ABTS ${ }^{+}$and $\mathrm{ROO} \bullet$ are more active than $\mathrm{DPPH} \bullet$, and it can react with a variety of amino acids (Prior et al., 2005). The $S$ atom of the Met side chain group also provides electrons, which can react with $\mathrm{ROO} \cdot$ in 
ORAC system and ABTS • ${ }^{+}$in ABTS system. Electrons was easier to react with $\mathrm{ROO} \cdot$ and showed higher antioxidant capacity in ORAC system than in ABTS system.

The discovery of Mora-Obandoet al. (Mora-Obando et al., 2014) demonstrated that there was synergistic effect between two subtypes of phospholipases $\mathrm{A}_{2}$, which could enhance the possibilities of $\mathrm{Ca}^{2+}$ entrances and the rate of the cell death when acting together upon C2C12 myotubes. During our study, we found that the combination of nonantioxidant amino acids and high antioxidant amino acids can significantly increase their total antioxidant capacities, while the combination of high antioxidant amino acids and weak antioxidant amino acids can have a slight or even no increase in their total antioxidant capacities. In DPPH study, Cys was combined with acidic amino acids, and the amide amino acids represented synergistic effects with ansignificantly increase of total antioxidant capacity. However when Cys was combined with hydroxy amino acids, it represented antagonism effects with a significant decrease of total antioxidant capacity. K Uchida et al. (K Uchida et al.,) had found that the antioxidative activity of histidine-containing peptides were higher than that of histidine itself, the effect of ortho-amino acid residues on the antioxidant activity of dipeptides is also of great significant. This may be related to the hydrophobicity, space volume, hydrogen bond, and electrical properties of these amino acid residues, in DPPH and ABTS studies, we found that Cys combined with other non-antioxidate exhibited different degrees of antioxidant capacity, which may have same reason with K Uchida had discovered. Both Glu and Asp are acidic amino acids, so they had similar antioxidant activity when combined with other high antioxidant amino acids, which showed that synergistic antioxidant activity is also related to the property of amino acids. Wang et al. (Wang et al., 2016b) found that the combination of serine and seleno-compound can significantly increase the GPx level, and all the concentrations of SelP and GPx increased with serine, SelP and GPx concentrations in adequate-Se group were significantly higher than the group with lowSe. During this study, Ser combined with Trp, Tyr and Cys gave a rise to the significantly synergistic effect in the ABTS radical scavenging activity assay, while Ser combined with Cys, His and Tyr led to the synergistic effect in the ORAC radical absorbance capacity assay.

Wiriyaphan et al. (Wiriyaphan et al., 2013) found out that the smaller peptides showed the higher antioxidant activities during the research. Therefore after comparing our results of the research with theirs, we draw the conclusion that which kinds of the amino acids have higher antioxidant capacities, smaller peptides or larger ones, that we need the further study.

\section{CONCLUSIONS}

In this article, the effects of 20 amino acids were included. Many amino acids quenched the ABTS radicalseffectively, while only $\mathrm{DPPH}^{+}$could be quenched by Cys. Amino acids can react with free radicals in antioxidant systems, thus reflecting the ability of free radical scavenging. In different antioxidant systems, the free radical scavenging ability of amino acids is also different. In this study, the results of DPPH and FRAP were similar, Cys had high antioxidant activity, all other amino acids showed no or very little antioxidant activity using the DPPH and FRAP method. In the ABTS and ORAC systems, an abundance of amino acids showed antioxidant activity, and the high antioxidant amino acids were Trp, Tyr and Cys, its antioxidant activities in ABTS and ORAC systems are very high, which indicates that the antioxidant activity of amino acids is not only related to amino acids but also to antioxidant systems. The antioxidant capacity of amino acids varies inversely as the combination of amino acids, this suggests that amino acid residues may affect hydrogen supply capacity and proton supply capacity of amino acids such as Tyr and Trp through spatial effects and hydrogen bond, etc. Amino acid have a great effect on synergistic antioxidant activity, which may be related to the hydrophobicity, spatial volume, hydrogen bond and electrical properties of the amino acids. The results obtained from this study showed that the antioxidant synergistic effect between the amino acids improved the antioxidant abilities of amino acids, and it was a common phenomenon during the reactions of amino acids. And the combination research in this study can also be applicable to inhibit the oxidative stress and it seems to be an excellent method to improve the effects of antioxidant.

\section{ACKNOWLEDGEMENTS}

This work was supported by National Natural Science Foundation of China (NO.31601486), the "13" ${ }^{\text {th }}$ FiveYear" science and technology project of The Education Department of Jilin Province (JJKH20170816KJ), the Jilin Scientific and Technological Development Program (20160622030JC), the Program for JLU Science and Technology Innovative Research Team (2017TD-29) and the Fundamental Research Funds for the Central Universities.

\section{REFERENCES}

Al-Gubory, K. H., F. Blachier, P. Faure and C. Garrel. 2016. Pomegranate peel extract decreases small intestine lipid peroxidation by enhancing activities of major antioxidant enzymes. J. Sci. Food Agric. 96: 3462-3468.

Balcerczyk, A., K. Sowa and G. Bartou. 2007. Metal chelators react also with reactive oxygen and nitrogen species. Biochem. Biophys. Res. Commun. 352: 522-525.

Benzie, I. F. and J. J. Strain. 1996. The ferric reducing ability of 
plasma (FRAP) as a measure of "antioxidant power": The FRAP assay. Anal. Biochem. 239: 70-76.

Bhattacharjee, A., S. K. Prasad, S. Pal, B. Maji, A. K. Syamal and S. Mukherjee. 2016. Synergistic protective effect of folic acid and vitamin B-12 against nicotine-induced oxidative stress and apoptosis in pancreatic islets of the rat. Pharm. Biol. 54: 433-444.

Cai, C., H. Miao, H. Qian, L. Yao, B. Wang and Q. Wang. 2016. Effects of industrial pre-freezing processing and freezing handling on glucosinolates and antioxidant attributes in broccoli florets. Food Chem. 210: 451-456.

Carocho, M. and I. Ferreira. 2013. A review on antioxidants, prooxidants and related controversy: Natural and synthetic compounds. screening and analysis methodologies and future perspectives. Food Chem. Toxicol. 51: 15-25.

Carocho, M., P. Morales and I. Ferreira. 2015. Natural food additives: Quo vadis? Trends Food Sci. Technol. 45: 284-295.

Damgaard, T., R. Lametsch and J. Otte. 2015. Antioxidant capacity of hydrolyzed animal by-products and relation to amino acid composition and peptide size distribution. J. Food Sci. Technol. Mysore. 52: 6511-6519.

Davalos, A., B. Bartolome and C. Gomez-Cordoves. 2004. Inhibition of methyl linoleate autoxidation by phenolics and other related compounds under mild oxidative conditions. J. Sci. Food Agric. 84: 631-638.

Liu, D., F. Pan, J. Liu, Y. Wang, T. Zhang, E. Wang and J. Liu. 2016. Individual and combined antioxidant effects of ginsenoside F2 and cyanidin-3-O-glucoside in human embryonic kidney 293 cells. RSC Adv. 6: 81092-81100.

Garrett, A. R., E. G. Weagel, A. D. Martinez, M. Heaton, R. A. Robison and K. L. O'Neill. 2014. A novel method for predicting antioxidant activity based on amino acid structure. Food Chem. 158: 490-496.

Hatano, B., D. Kubo and H. Tagaya. 2006. Study on the reactivity of diarylmethane derivatives in supercritical alcohols media: Reduction of diarylmethanols and diaryl ketones to diarylmethanes using supercritical 2-propanol. Chem. Pharm. Bull. 54: 1304-1307.

Hernández-Ledesma, B., A. Davalos, B. Bartolome and Amigo, L. 2005. Preparation of antioxidant enzymatic hydrolysates from alpha-lactalbumin and beta-lactoglobulin. Identification of active peptides by HPLC-MS/MS.[J]. J. Agric. Food Chem. 53: 588-593.

Ka, H., B. Yi, M. J. Kim and J. Lee. 2016. Evaluation of antioxidant or prooxidant properties of selected amino acids using in vitro assays and in oil-in-water emulsions under riboflavin sensitization. J. Food Sci. 81: C1118-C1123.

Liao, K. L. and M. C. Yin. 2000. Individual and combined antioxidant effects of seven phenolic agents in human erythrocyte membrane ghosts and phosphatidylcholine liposome systems: Importance of the partition coefficient. J. Agric. Food Chem. 48: 2266-2270.

Liu, R. H. 2003. Health benefits of fruit and vegetables are from additive and synergistic combinations of phytochemicals. Am. J. Clin. Nutr. 78: 517S-520S.

Liu, R. H. 2004. Potential synergy of phytochemicals in cancer prevention: Mechanism of action. J. Nutr. 134: 3479S-3485S.

Marcuse, R. 1960. Antioxidative effect of amino-acids. Nature. 186: 886-887.

Mora-Obando, D., J. Fernandez, C. Montecucco, J. M. Gutierrez and B. Lomonte. 2014. Synergism between Basic Asp49 and Lys49 Phospholipase $A(2)$ Myotoxins of viperid snake venom in vitro and in vivo. PLoS One. 9: e109846.

Nagaoka, S. I., A. Nitta, A. Suemitsu and K. Mukai. 2016. Tunneling effect in vitamin E recycling by green tea. RSC Adv. 6: 47325-47336.
Ou, B. X., D. J. Huang, M. Hampschl-Woodill and J. A. Flanagan. 2003. When east meets west: The relationship between yinyang and antioxidation-oxidation. FASEB J. 17: 127-129.

Palozza, P. and N. I. Krinsky. 1992. Beta-Carotene and alphatocopherol are synergistic antioxidants. Arch. Biochem. Biophys. 297: 184-187.

Re, R., N. Pellegrini, A. Proteggente, A. Pannala, M. Yang and C. Rice-Evans. 1999. Antioxidant activity applying an improved ABTS radical cation decolorization assay. Free Radic. Biol. Med. 26: 1231-1237.

Ren, Y., H. Wu, X. Li, F. Lai, G. Zhao and X. Xiao. 2014. A twostep, one-pot enzymatic method for preparation of duck egg white protein hydrolysates with high antioxidant activity. Appl. Biochem. Biotechnol. 172: 1227-1240.

Prior, R. L., X. Wu and K. Schaich. 2005. Standardized methods for the determination of antioxidant capacity and phenolics in foods and dietary supplements[J]. J. Agric. Food Chem. 53: 4290-302.

Robbins, K. S., P. Greenspan and R. B. Pegg. 2016. Effect of pecan phenolics on the release of nitric oxide from murine RAW 264.7 macrophage cells. Food Chem. 212: 681-687.

Rossetto, M., P. Vanzani, F. Mattivi, M. Lunelli, M. Scarpa and A. Rigo. 2002. Synergistic antioxidant effect of catechin and malvidin 3-glucoside on free radical-initiated peroxidation of linoleic acid in micelles. Arch. Biochem. Biophys. 408: 239-245.

Saoudi, S., N. Chammem, I. Sifaoui, M. Bouassida-Beji, I. A. Jimenez, I. L. Bazzocchi, S. D. Silva, M. Hamdi and M. R. Bronze. 2016. Influence of Tunisian aromatic plants on the prevention of oxidation in soybean oil under heating and frying conditions. Food Chem. 212: 503-511.

Triantis, T. M., E. Yannakopoulou, A. Nikokavoura, D. Dimotikali and K. Papadopoulos. 2007. Chemiluminescent studies on the antioxidant activity of amino acids[J]. Anal. Chim. Acta. 591: 106-111.

Uchida, K. and S. Kawakishi. 1992. Sequence-dependent reactivity of histidine-containing peptides with copper(II)/ascorbate[J]. J. Agric. Food Chem. 40: 13-16.

Vavrusova, M., H. Pindstrup, L. B. Johansen, M. L. Andersen, H. J. Andersen and L. H. Skibsted. 2015. Characterisation of a whey protein hydrolysate as antioxidant. Int. Dairy J. 47: 86-93.

Wang, C., B. Wang and B. Li. 2016a. Bioavailability of peptides from casein hydrolysate in vitro: Amino acid compositions of peptides affect the antioxidant efficacy and resistance to intestinal peptidases. Food Res. Int. 81: 188-196.

Wang, Q., L. C. Sun, Y. Q. Liu, J. X. Lu, F. Han and Z. W. Huang. 2016b. The synergistic effect of serine with selenocompounds on the expression of SelP and GPx in HepG2 Cells. Biol. Trace Element Res. 173: 291-296.

Wiriyaphan, C., B. Chitsomboon, S. Roytrakul and J. Yongsawadigul. 2013. Isolation and identification of antioxidative peptides from hydrolysate of threadfin bream surimi processing byproduct. J. Func. Foods. 5: 1654-1664.

Wright, J. S., E. R. Johnson and G. A. Dilabio. 2001. Predicting the activity of phenolic antioxidants:ctivity of phemethod, analysis of substituent effects, and application to major families of antioxidants[J]. J. Am. Chem. Soc. 123: 1173.

Yang, J. and R. H. Liu. 2009. Synergistic effect of apple extracts and quercetin 3-beta-D-glucoside combination on antiproliferative activity in MCF-7 human breast cancer cells in vitro. J. Agric. Food Chem. 57: 8581-8586.

Zheng, L., H. Dong, G. Su, Q. Zhao and M. Zhao. 2016. Radical scavenging activities of Tyr-, Trp-, Cys- and Met-Gly and their protective effects against AAPH-induced oxidative damage in human erythrocytes. Food Chem. 197: 807-813. 